\title{
Strategic Plan for Nuclear Energy - Knowledge Base for Advanced Modeling and Simulation (NE-KAMS)
}

Kimberlyn C. Mousseau

Richard W. Johnson

Hyung Lee

September 2011

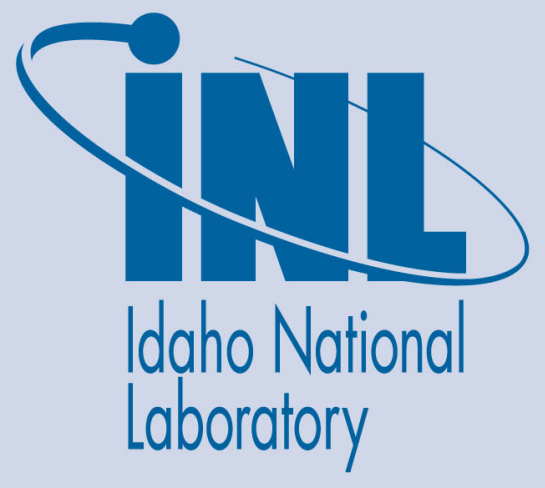

The INL is a U.S. Department of Energy National Laboratory operated by Battelle Energy Alliance 
INL/EXT-11-23415

FCRD-NEAMS-2011-000454

\title{
Strategic Plan for Nuclear Energy - Knowledge Base for Advanced Modeling and Simulation (NE-KAMS)
}

${ }^{1}$ Bettis Laboratory

\author{
Kimberlyn C. Mousseau \\ Richard W. Johnson \\ Hyung Lee ${ }^{1}$
}

September 2011

\begin{abstract}
Idaho National Laboratory
Idaho Falls, Idaho 83415
\end{abstract}

http://www.inl.gov

\author{
Prepared for the \\ U.S. Department of Energy \\ Office of Nuclear Energy \\ Under DOE Idaho Operations Office \\ Contract DE-AC07-05ID14517
}




\section{EXECUTIVE SUMMARY}

The US Department of Energy, Office of Nuclear Energy (DOE-NE), has been tasked with the important mission of ensuring that nuclear energy remains a compelling and viable energy source in the U.S. The motivations behind this mission include cost-effectively meeting the expected increases in the power needs of the country, reducing carbon emissions, and reducing dependence on foreign energy sources. In the near term, to ensure that nuclear power remains a key element of U.S. energy strategy, the DOE-NE will be working with the nuclear industry to support safe and efficient operations of existing nuclear power plants. In the long term, the DOE-NE will be investing in research and development (R\&D) and working in concert with the nuclear industry to build new, safer and more efficient nuclear power plants. The safe and efficient operations of existing nuclear power plants and designing and licensing new reactor designs, however, will require the extensive use of advanced modeling and simulation (M\&S). Thus, it is expected that M\&S will play a key role in ensuring the safe and efficient operation of existing and new nuclear reactors.

The complexity of nuclear reactor power plants, as well as the cost and difficulty associated with testing nuclear reactor systems makes the use of $\mathrm{M} \& \mathrm{~S}$ a desirable tool for nuclear reactor design, analysis and licensing. Thus, engineering analysis and performance characterization of existing and new reactor designs will employ advanced M\&S tools, such as computational fluid dynamics (CFD) and computational structural mechanics (CSM), in addition to the traditional thermal hydraulics $(\mathrm{T} / \mathrm{H})$ and systems analysis codes. The DOE-NE, in fact, has been actively developing and promoting the use of advanced M\&S in reactor design and analysis through its R\&D programs, e.g., the Nuclear Energy Advanced Modeling and Simulation (NEAMS) and Consortium for Advanced Simulation of Light Water Reactors (CASL) programs. Also, nuclear reactor vendors are already using CFD and CSM, for design, analysis, and licensing. However, these M\&S tools cannot be used with confidence for nuclear reactor applications unless supported by verification and validation (V\&V) and uncertainty quantification (UQ) which provide quantitative measures of uncertainty for specific applications.

$\mathrm{V} \& \mathrm{~V}$ and UQ are the primary means to assess the accuracy and reliability of M\&S and, hence, to establish confidence in M\&S. Though the nuclear industry has established standards and processes for carrying out V\&V and UQ for systems analysis codes and simulations, at present, similar standards and processes for high fidelity M\&S tools such as CFD have not reached the same level of maturity. However, the nuclear industry recognizes that such standards and processes are needed and that the resources required to support V\&V and UQ for CFD for nuclear applications is significant. In fact, no single organization, whether a commercial company or government laboratory, has the resources required to organize, develop and maintain the needed V\&V and UQ program. What is needed is a standardized program for V\&V and UQ at a national or even international level, with a consortium of partners from government, academia and industry. Specifically, what is needed is a structured knowledge base that collects, evaluates and stores verification and validation data, and shows how it can be used to perform $\mathrm{V} \& \mathrm{~V}$ and UQ. This knowledge base can promote collaboration and provide for sharing of resources to support engineering and licensing applications.

The Nuclear Energy Knowledge base for Advanced Modeling and Simulation (NE-KAMS) is being developed at the Idaho National Laboratory in conjunction with Bettis Laboratory, Sandia National Laboratories, Argonne National Laboratory, Utah State University and others. The 
objective of this consortium is to establish a comprehensive and web-accessible knowledge base to provide $\mathrm{V} \& \mathrm{~V}$ and $\mathrm{UQ}$ resources for $\mathrm{M} \& \mathrm{~S}$ for nuclear reactor design, analysis and licensing. The knowledge base will serve as an important resource for technical exchange that will enable credible computational models and simulations for application to nuclear power. NE-KAMS will serve as a valuable resource for the nuclear industry, academia, the national laboratories, the U.S. Nuclear Regulatory Commission (NRC), and the public, and will help ensure the safe, economical and reliable operation of existing and future nuclear reactors.

Existing V\&V databases, such as the European Research Community on Flow, Turbulence and Combustion (ERCOFTAC), the European QNET-CFD database, and the U.S. NPARC Alliance database, serve only as repositories for V\&V cases. The NE-KAMS knowledge base will provide guidance for progressive levels of $\mathrm{V} \& \mathrm{~V}$ completeness assessment of $\mathrm{V} \& \mathrm{~V}$ benchmarks and best practices for application of M\&S. Specifically, the NE-KAMS knowledge base will assist analysts, model developers, experimentalists, designers, and regulators by:

- Establishing accepted standards, requirements and best practices for V\&V and UQ of computational models and simulations,

- Establishing accepted standards and procedures for assessing experimental and numerical benchmark data,

- Providing readily accessible databases with long-term storage for code verification and validation benchmark data that can be used in V\&V assessments and methods development,

- Providing a searchable knowledge base of information, documents and data on V\&V, and

- Providing web-enabled tools and utilities for V\&V and UQ activities, data assessment and processing, and information and data searches.

From its inception, NE-KAMS will directly support nuclear energy R\&D programs within the U.S. Department of Energy (DOE), including CASL, NEAMS, Light Water Reactor Sustainability (LWRS), Small Modular Reactors (SMR), and Next Generation Nuclear Power Plant (NGNP). These programs all involve M\&S for nuclear reactor systems and it is envisioned that NE-KAMS will facilitate collaboration and sharing of resources and expertise across these programs. The initial focus for the NE-KAMS effort will be on supporting the use of CFD for simulation of nuclear reactor systems, but will eventually expand to include, materials, fuel system performance and other areas of M\&S as time and funding allow.

\section{Primary Elements of NE-KAMS}

The primary elements of the NE-KAMS knowledge base designed to support computational $\mathrm{M} \& \mathrm{~S}$ in nuclear energy sciences and engineering are listed below.

Element 1. Standards, requirements and best practices for performing V\&V and UQ assessments in establishing credibility in computational analyses of nuclear reactor systems.

Element 2. Standards and procedures for the evaluation and classification of experimental and numerical benchmark data for use in V\&V assessments.

Element 3. Quality-assessed, web-accessible databases for nuclear related experimental and numerical benchmark data and attendant metadata that can be used in V\&V assessments of computational models and simulations and methods development. 
Element 4. A knowledge base of searchable information, documents and data related to V\&V and UQ of computational models and simulations, including examples of credible V\&V assessment and uncertainty analysis.

Element 5. Targeted V\&V case studies of nuclear reactor systems, components and processes to enable reduction in uncertainty and improvement in applicability of computational models and simulations used in the nuclear industry.

\section{NE-KAMS Implementation Strategy}

Initially, the NE-KAMS effort will work on the implementation of all of the primary elements of the knowledge base. A graded approach will be followed in which preliminary but useful progress will be made for each element. With time, each element will be improved, revised and expanded as the development processes evolve and lessons learned are incorporated. This approach will also provide useful information and resources early on in the development of the knowledge base, especially for the DOE nuclear energy M\&S programs identified above.

The initial focus for the NE-KAMS effort will be on supporting the use of CFD for M\&S of nuclear reactor systems. CFD validation datasets, both existing and legacy experimental data, will be sought first for inclusion in the NE-KAMS knowledge base. In addition, targeted CFD $\mathrm{V} \& \mathrm{~V}$ case studies will be performed to aid in the development of guidelines and recommended practices for reducing uncertainty and improving applicability of thermal hydraulics $(\mathrm{T} / \mathrm{H})$ analysis models and methods being used in the nuclear industry.

Once the NE-KAMS knowledge base has reached a sufficient level of functionality for CFD and $\mathrm{T} / \mathrm{H}$, the same graded approach will be applied to develop and implement additional databases that are pertinent to nuclear reactor design and safety analysis, such as the materials and fuel performance databases.

\section{NE-KAMS Implementation and Operations}

The NE-KAMS knowledge base will maximize the benefits to its users by providing the means to access rapidly and coherently the required information and data and efficiently and effectively utilize them in support of $\mathrm{V} \& \mathrm{~V}$ and $\mathrm{UQ}$ assessments and methods development. To that end, NE-KAMS will deploy state-of-the-art technology in information management systems and web services to leverage high performance computing and web technologies. The computing infrastructure will include knowledge base systems software, web-based user interface systems, high performance computing and storage systems, and high performance networks. Webenabled applications, tools and utilities for V\&V and UQ activities, data access and processing, and information and data searches will be developed, implemented and deployed.

The NE-KAMS approach to computing will be unique in that the users can interact with the knowledge base and perform tasks that would have to be done offline with conventional databases, which typically only allow for downloading of information and data. It is envisioned that with the full deployment of the NE-KAMS knowledge base, much, if not all, of the V\&V and UQ tasks of the user will be performed in the NE-KAMS knowledge base. It is thus argued that the NE-KAMS knowledge base, when fully implemented and deployed, will bring the power of high performance computing to the masses in that there will no longer be technical or 
financial barriers for accessing the powers of high performance computing for NE-KAMS knowledge base users.

The details of the NE-KAMS knowledge base and its implementation will be provided in two NE-KAMS planning documents:

- NE-KAMS Knowledge Base High Level Requirements

- NE-KAMS Knowledge Base Code Verification and Validation Data Standards and Requirements 


\section{TABLE OF CONTENTS}

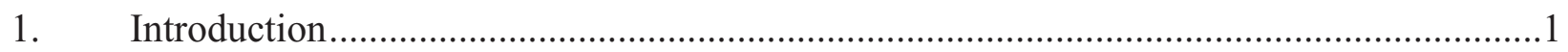

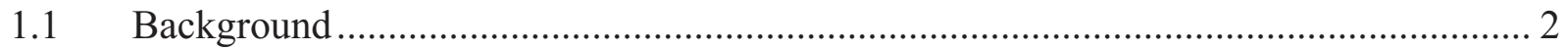

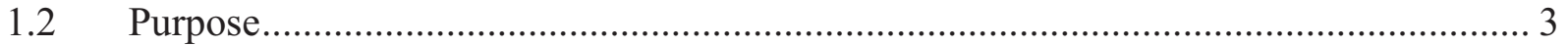

$1.3 \quad$ Scope

2. Description of the NE-KAMS Knowledge Base ................................................................

2.1 Motivations, Objectives and Elements ……………................................................... 4

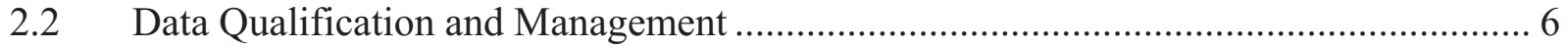

2.3 Data Standards Clearing Committee ....................................................................... 7

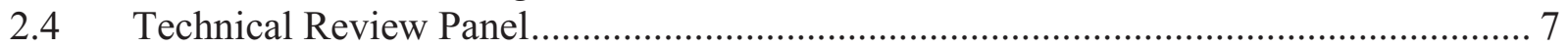

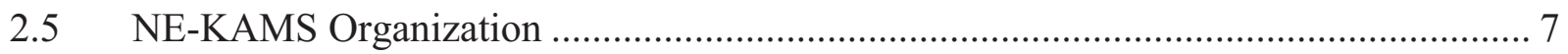

3. Verification and Validation of CFD for Nuclear Reactor Applications .............................9

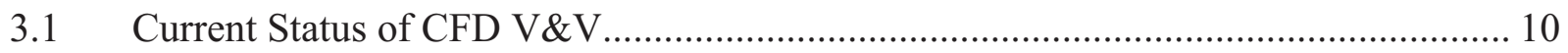

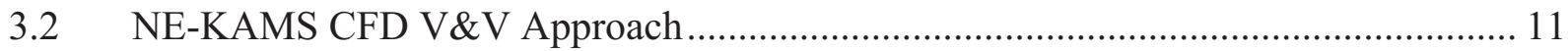

3.3 Precedents from Nuclear Reactor Physics ............................................................... 12

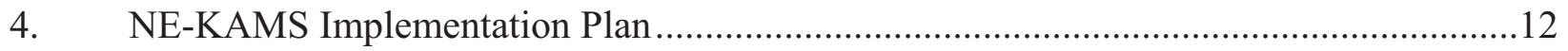

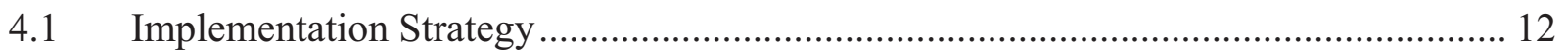

4.2 Implementation Activities................................................................................. 13

5. NE-KAMS Implementation and Operations ..................................................................

5.1 NE-KAMS Standards, Databases and Infrastructure .................................................. 16

$5.2 \quad$ NE-KAMS Challenges and Issues ..................................................................... 18

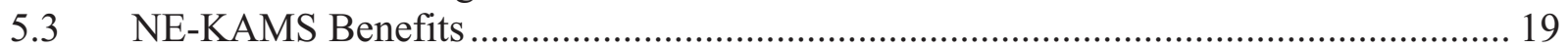

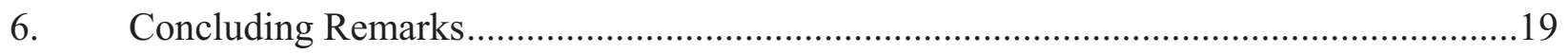

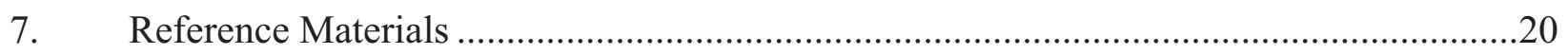

APPENDIX A NE-KAMS WORKING GROUP AND SUB-GROUPS ……………………....21

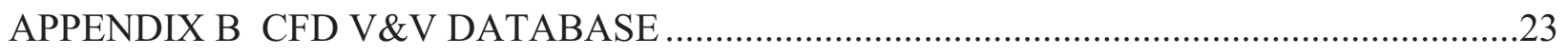

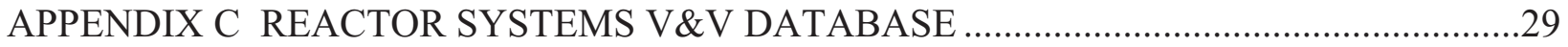




\section{ACRONYMS}

ANL Argonne National Laboratory

CASL Consortium for Advanced Simulation of Light Water Reactors

CFD computational fluid dynamics

CSAU code scaling, applicability and uncertainty

CSM computational structural mechanics

DOE Department of Energy

DOE-NE Department of Energy - Office of Nuclear Energy

EPRI Electric Power Research Institute

INL Idaho National Laboratory

LWRS Light Water Reactor Sustainability

M\&S modeling and simulation

NE-KAMS Nuclear Energy Knowledge base for Advanced Modeling and Simulation

NEAMS Nuclear Energy Advanced Modeling and Simulation

NGNP Next Generation Nuclear Plant

NNSA National Nuclear Security Administration

NRC Nuclear Regulatory Commission

R\&D research and development

SMR Small Modular Reactors

SNL Sandia National Laboratories

$\mathrm{T} / \mathrm{H} \quad$ thermal hydraulics

UQ uncertainty quantification

USU Utah State University

$\mathrm{V} \& \mathrm{~V} \quad$ verification and validation 


\section{Introduction}

The US Department of Energy, Office of Nuclear Energy (DOE-NE), has been tasked with the important mission of ensuring that nuclear energy remains a compelling and viable energy source in the U.S. The motivations behind this mission include cost-effectively meeting the expected increases in the power needs of the country, reducing carbon emissions, and reducing dependence on foreign energy sources. In the near term, to ensure that nuclear power remains a key element of U.S. energy strategy, the DOE-NE will be working with the nuclear industry to support safe and efficient operations of existing nuclear power plants. In the long term, the DOE-NE will be investing in research and development (R\&D) and working in concert with the nuclear industry to build new, safer and more efficient nuclear power plants. The safe and efficient operations of existing nuclear power plants and designing and licensing new reactor designs, however, will require the extensive use of advanced modeling and simulation (M\&S). Thus, it is expected that M\&S will play a key role in ensuring the safe and efficient operation of existing and new nuclear reactors.

The complexity of nuclear reactor power plants, as well as the cost and difficulty associated with testing nuclear reactor systems makes the use of $\mathrm{M} \& \mathrm{~S}$ a desirable tool for nuclear reactor design, analysis and licensing. Thus, engineering analysis and performance characterization of existing and new reactor designs will employ advanced M\&S tools, such as computational fluid dynamics (CFD) and computational structural mechanics (CSM), in addition to the traditional thermal hydraulics $(\mathrm{T} / \mathrm{H})$ and systems analysis codes. The DOE-NE, in fact, has been actively developing and promoting the use of advanced M\&S in reactor design and analysis through its R\&D programs, e.g., the Nuclear Energy Advanced Modeling and Simulation (NEAMS) and Consortium for Advanced Simulation of Light Water Reactors (CASL) programs. Also, nuclear reactor vendors are already using CFD and CSM, for design, analysis, and licensing. However, these $M \& S$ tools cannot be used with confidence for nuclear reactor applications unless supported by verification and validation (V\&V) and uncertainty quantification (UQ) which provide quantitative measures of uncertainty for specific applications.

$\mathrm{V} \& \mathrm{~V}$ and UQ are the primary means to assess the accuracy and reliability of M\&S and, hence, to establish confidence in M\&S. Though the nuclear industry has established standards and processes for carrying out V\&V and UQ for systems analysis codes and simulations, at present, similar standards and processes for high fidelity M\&S tools such as CFD have not reached the same level of maturity. However, the nuclear industry recognizes that such standards and processes are needed and that the resources required to support V\&V and UQ for CFD for nuclear applications is significant. In fact, no single organization, whether a commercial company or government laboratory, has the resources required to organize, develop and maintain the needed V\&V and UQ program. What is needed is a standardized program for V\&V and UQ at a national or even international level, with a consortium of partners from government, academia and industry. Specifically, what is needed is a structured knowledge base that collects, evaluates and stores verification and validation data, and shows how it can be used to perform $\mathrm{V} \& \mathrm{~V}$ and UQ. This knowledge base can promote collaboration and provide for sharing of resources to support engineering and licensing applications.

The Nuclear Energy Knowledge base for Advanced Modeling and Simulation (NE-KAMS) is being developed at the Idaho National Laboratory in conjunction with Bettis Laboratory, Sandia 
National Laboratories, Argonne National Laboratory, Utah State University and others. The objective of this consortium is to establish a comprehensive and web-accessible knowledge base to provide $\mathrm{V} \& \mathrm{~V}$ and $\mathrm{UQ}$ resources for $\mathrm{M} \& \mathrm{~S}$ for nuclear reactor design, analysis and licensing. The knowledge base will serve as an important resource for technical exchange that will enable credible computational models and simulations for application to nuclear power. NE-KAMS will serve as a valuable resource for the nuclear industry, academia, the national laboratories, the U.S. Nuclear Regulatory Commission (NRC), and the public, and will help ensure the safe, economical and reliable operation of existing and future nuclear reactors.

The NE-KAMS Strategic Plan describes the mission, goals and intended uses of the NE-KAMS knowledge base and a plan for its implementation. It describes the technical drivers that led to the development of the knowledge base and the DOE-NE research, development and demonstration programs that NE-KAMS supports. It outlines the near and long term objectives of the NE-KAMS effort and describes the approaches that will be taken to develop, implement, operate and maintain the NE-KAMS knowledge base. It also describes the key elements of NEKAMS that set it apart from previously developed V\&V databases. In addition, it describes approaches that will be taken to implement these elements which will ensure that the NE-KAMS knowledge base remains a viable resource for assuring the credibility of M\&S for nuclear reactor design and safety analysis applications.

\subsection{Background}

Over the past decade, there has been a worldwide increase in the use of M\&S for the design and analysis of nuclear reactors. M\&S provides a more affordable approach to analyzing a nuclear reactor than traditional testing and demonstration; it also provides much more information about the detailed physics of the system. With the advent of high performance computing and advanced modeling techniques, government agencies, industry and academia are using M\&S as a means to explore and assess the performance of nuclear reactors and related systems. The DOE$\mathrm{NE}$, for example, is investing in these capabilities by formally establishing national M\&S research, development and demonstration programs. One is called the Consortium for Advanced Simulation of Light Water Reactors (CASL), whose primary objective is to establish numerical simulation as a primary means for the determination of nuclear reactor system behavior and performance. Others include the Nuclear Energy Advanced Modeling and Simulation (NEAMS), the Light Water Reactor Sustainability (LWRS), the Small Modular Reactors (SMR), and the Next Generation Nuclear Power Plant (NGNP) programs.

Today M\&S is being used extensively to understand the behavior and predict the performance of nuclear reactors for normal operating conditions as well as for numerous accident scenarios. For M\&S to be credible, however, the M\&S calculations, including the physical models used, e.g., for simulating turbulence, heat transfer, multiphase flow, chemical reactions, etc., must be validated against experimental data to ensure the level of accuracy needed and expected for their intended use. NE-KAMS will be a comprehensive and web-accessible knowledge base for nuclear energy sciences and engineering which will support quality assurance processes for the use of computational fluid dynamics (CFD) and other computational physics methods in nuclear reactor systems design and safety analysis. It is envisioned that NE-KAMS will serve as an important resource for technical exchange and collaboration for the researchers and analysts in industry, government and academia. NE-KAMS will directly aid in establishing accepted standards, requirements and best practices for $\mathrm{V} \& \mathrm{~V}$ of computational models and simulations 
and providing readily accessible databases for nuclear energy related experimental and numerical benchmark data that can be used in V\&V assessments and computational methods development.

The nuclear industry arguably has the most stringent and technically sophisticated regulatory body that carefully scrutinizes analysis results as well as the codes and tools used for such analysis. A means by which the U.S. Nuclear Regulatory Commission (NRC) assesses or qualifies any licensing calculation performed using $M \& S$ is by comparing the $M \& S$ results to applicable experimental data, similar to V\&V assessments. Thus, the NE-KAMS knowledge base could become an important resource not only for nuclear reactor design and safety researchers and analysts, but also for the regulators in establishing confidence in computational modeling and simulations for nuclear reactor design and safety analysis applications.

\subsection{Purpose}

The purpose of this strategic plan is to provide the DOE-NE M\&S programs with a high-level description and intended use of the NE-KAMS knowledge base. Initially the plan will focus on addressing the $\mathrm{V} \& \mathrm{~V}$ and uncertainty quantification (UQ) requirements and needs of the DOENE programs CASL, NEAMS, LWRS, SMR and NGNP. The stated DOE-NE research and development $(\mathrm{R} \& \mathrm{D})$ objectives are given below:

"The DOE-NE 2010 Research and Development (R\&D) Roadmap has organized its activities in accordance with four objectives that ensure nuclear energy remains a compelling and viable energy option for the US. The objectives are 1) develop technologies and other solutions that can improve the reliability, sustain the safety, and extend the life of the current reactors, 2) develop improvements in the affordability of new reactors to enable nuclear energy to help meet the Administration's energy security and climate change goals, 3) develop and sustain nuclear fuel cycles, and 4) understand and minimize the risks of nuclear proliferation and terrorism.",

It is envisioned that NE-KAMS will help the DOE-NE achieve its R\&D objectives by coordinating and facilitating collaboration and sharing of resources and expertise for $\mathrm{V} \& \mathrm{~V}$ and UQ across DOE-NE M\&S programs. In the longer term, the plan outlines the approaches and steps that will be taken to expand the capabilities and scope of NE-KAMS and ensure that NEKAMS remains a viable resource for assuring the credibility of computational M\&S for nuclear reactor design and safety analysis applications.

\subsection{Scope}

This strategic plan details both the short- and long-term expectations for NE-KAMS. In the near term, NE-KAMS will provide direct support for V\&V and UQ activities and processes of DOENE M\&S programs and for capturing and preserving the knowledge gained from the various DOE-NE M\&S programs, including applicable experimental and numerical data. Specific details on how NE-KAMS will support the DOE-NE M\&S programs are as follows:

\footnotetext{
${ }^{1}$ Objective 1: Extend Life, Improve Performance, and Maintain Safety of the Current Fleet Implementation Plan, U.S. Department of Energy, Office of Nuclear Energy, January 2011.
} 
- NEAMS - NE-KAMS is an integral part of the NEAMS Program and, as such, it will attempt to support all the $V \& V$ activities and processes of NEAMS. Initially, NE-KAMS will focus on supporting the CFD component of NEAMS.

- CASL - In the near term, NE-KAMS will focus on supporting the V\&V activities and processes for the use of CFD in the CASL Program: single- and two-phase flows and fluid structure interaction. In the longer term, NE-KAMS will provide support for chemical-kinetics, materials and fuel system performance.

- LWRS - NE-KAMS will focus on supporting the V\&V activities and processes for the use of next generation reactor systems code R7 and related thermal hydraulics $(\mathrm{T} / \mathrm{H})$ analysis codes in the LWRS Program (see Appendix C).

- SMR - NE-KAMS will focus on supporting the V\&V activities and processes for the use of CFD in the SMR Program. It is expected that CFD will be used extensively in the SMR Program to assess the behavior and performance of integral SMR designs where the reactor systems are tightly coupled to the containment.

- NGNP - NE-KAMS will focus on capturing and preserving the NGNP Program knowledge and data.

As the DOE-NE M\&S programs continue to grow, a collaborative approach to the integration of these programs will be undertaken along with a systematic review and revision of this plan to ensure that the full potential of NE-KAMS is utilized. In addition, it is expected that NE-KAMS, through its focused development activities and $\mathrm{V} \& \mathrm{~V}$ case studies, will support the use of computational $\mathrm{M} \& \mathrm{~S}$ in the nuclear industry by developing guidelines and recommended practices aimed at reducing uncertainty and improving applicability of existing analysis methods. In the longer term, the plan describes the approaches and steps that will be taken to expand the capabilities and scope of NE-KAMS to support a broader user community, not only in the nuclear energy sciences and engineering, but also users in other disciplines and industries.

\section{Description of the NE-KAMS Knowledge Base}

The mission of NE-KAMS is as follows:

The mission of NE-KAMS is to provide leadership through the creation of verification and validation data standards and a community accessible knowledge base to enable credible, predictive modeling and simulation for nuclear energy applications.

In this section, the motivations, objectives and elements for NE-KAMS along with its organization are described.

\subsection{Motivations, Objectives and Elements}

The overall objective for the establishment of the NE-KAMS knowledge base is to support the use of M\&S for nuclear reactor applications, including design, analysis and licensing calculations that will help ensure the safe, economic and reliable operation of existing and future nuclear reactors. The motivations and objectives for the creation of NE-KAMS include the following: 
- Promote the culture of quality and accuracy in computational sciences and engineering by developing and establishing accepted standards, requirements, and procedures for performing V\&V and UQ of computational models and simulations that can be followed and adhered to by researchers, developers and analysts; such standards and procedures will help educate current and future generations of scientists and engineers in the nuclear energy sciences and engineering fields.

- Create a central repository and exchange for experimental and numerical benchmark data and supporting metadata to ensure that the body of knowledge gained from the DOE-NE $\mathrm{M} \& \mathrm{~S}$ programs is and will continue to be accessible to stakeholders in industry, government and academia.

- Share the cost of developing and implementing a knowledge base for nuclear energy sciences and engineering among the stakeholders and interested parties in industry, government and academia, including nuclear power utilities, EPRI, nuclear reactor vendors, DOE national laboratories, universities, and the U.S. NRC.

- Provide readily accessible, web-enabled tools and utilities to maximize the benefits of a knowledge base for nuclear energy sciences and engineering to its users in industry, government and academia.

Unlike most $\mathrm{V} \& \mathrm{~V}$ databases which just serve as repositories for $\mathrm{V} \& \mathrm{~V}$ cases and documents, the NE-KAMS knowledge base will provide value-added V\&V, UQ and computational methods development technical support services to its users. This will be accomplished by (a) establishing standards, requirements and best practices for V\&V and UQ assessments, (b) establishing standards and procedures for qualifying and classifying experimental and numerical benchmark data, (c) providing readily accessible databases for experimental and numerical benchmark data for $\mathrm{V} \& \mathrm{~V}$ assessments, (d) providing a searchable knowledge base of information, documents and data, and (e) providing web-enabled tools and utilities for $\mathrm{V} \& \mathrm{~V}$ activities. The elements of the NE-KAMS knowledge base are the following:

- Standards, requirements and best practices for performing V\&V and UQ assessments in establishing credibility in computational analyses of nuclear reactor systems, components and processes;

- Standards and procedures for the evaluation and classification of experimental and numerical benchmark data for use in $\mathrm{V} \& \mathrm{~V}$ assessments, which will characterize the quality of the data used in $\mathrm{V} \& \mathrm{~V}$ assessments via completeness categories, and provide clear guidelines on what is required to generate high-quality $\mathrm{V} \& \mathrm{~V}$ benchmark data by highlighting the importance of several key completeness categories;

- Quality-assessed, web-accessible databases for nuclear energy related experimental and numerical benchmark data and attendant metadata that can be used in V\&V assessments of computational models and simulations and computational methods development; metadata includes information about the experiment such as data uncertainty analyses, instrumentation used, the as-tested geometry and the experimental procedures followed to obtain the data;

- A knowledge base of searchable information, documents and data related to V\&V and UQ of computational models and simulations, including examples of credible V\&V assessment and uncertainty analysis; 
- Targeted V\&V case studies of nuclear reactor systems, components and processes to enable reduction in uncertainty and improvement in applicability of computational models and simulations used in the nuclear industry;

- Validation pyramids and Phenomena Identification and Ranking Tables (PIRT charts) for applicable nuclear reactor designs and systems;

- Development and implementation of the knowledge base system software, hardware and facility infrastructure for data collection, processing, qualification, storage and utilization with the goal of retaining and maximizing access to both the data and the metadata;

- Web-enabled applications, tools and utilities for V\&V and UQ activities, data assessment and processing, and information and data searches.

Additional details on the NE-KAMS knowledge base elements and their implementation will be provided in the two NE-KAMS planning documents:

- NE-KAMS Knowledge Base High Level Requirements

- NE-KAMS Knowledge Base Code Verification and Validation Data Standards and Requirements

\subsection{Data Qualification and Management}

The experimental data obtained from validation quality experiments requires a high level of fidelity, resolution and documentation, including a complete and thorough description of the boundary and initial conditions, the instrumentation used and the experimental procedures followed, the as-tested geometry of the validation experiment, and an appropriate uncertainty analysis of the experimental data. It is intended to develop standards and qualify datasets that are submitted to NE-KAMS against these standards. A set of qualification procedures to be performed against all entering data will be developed and documented prior to accepting data into the database. These qualification procedures will then be applied to candidate data for inclusion in the database. It is expected that the data qualification standards and procedures will assess the following:

- Data provenance;

- Metadata included:

$\circ$ uncertainty analysis,

○ instrumentation used,

○ experimental procedures,

$\circ$ as-tested geometry,

- measurement of boundary and initial conditions;

- Assessment of the relevance of the data to specific reactor applications;

- Adherence to established data quality standards (NQA-1 and ISO 9001);

- Adherence to NE-KAMS data standards;

- Detailed error estimation for code verification benchmarks. 
A data management plan will be developed to define the management procedures needed to collect, handle and store the data. This plan will specifically describe all of the procedures that will be implemented to manage the database, including specific practices that will be applied to the data, e.g., data security requirements.

\subsection{Data Standards Clearing Committee}

A data standards clearing committee will be established to ensure that the data qualification process has been completed per NE-KAMS policies and procedures. The committee is expected to meet at least annually with the specific and ongoing responsibilities to:

- Review the data quality objectives and ensure that the data that are entered into NE-KAMS meet the objectives;

- Conduct a preliminary review of data submitted for inclusion in NE-KAMS;

- Review standards for qualifying data submitted to NE-KAMS and standards for generation of new V\&V data;

- Approve proposed revisions to data qualification and generation standards.

\subsection{Technical Review Panel}

The Technical Review Panel will consist of representatives from industry, government and academia. Initially, the Technical Review Panel will primarily consist of experimental and computational experts that can guide and inform the development of the desired standards and processes. The Technical Review Panel will provide

- Technical critique/advice;

- Help to ensure that deliverables are appropriate;

- Representation of users/customers/programs;

- Academia/Industry perspectives.

Once the standards and processes for NE-KAMS have been established, a Technical Advisory Panel will take the place of the Technical Review Panel. The Technical Advisory Panel will be comprised of members from DOE national laboratories, government agencies and nuclear energy industries. Where possible, members who are already participating in one of the major DOE M\&S programs will be selected.

\subsection{NE-KAMS Organization}

Schematics are provided below that show the NE-KAMS knowledge base elements and organization. Figure 1 illustrates the elements of the NE-KAMS knowledge base and Figure 2 provides a diagram of the NE-KAMS organization, including the stakeholders, committees, organizing group and working subgroups. Appendix A provides a detailed list of working group members and sub-groups that will address particular elements. 


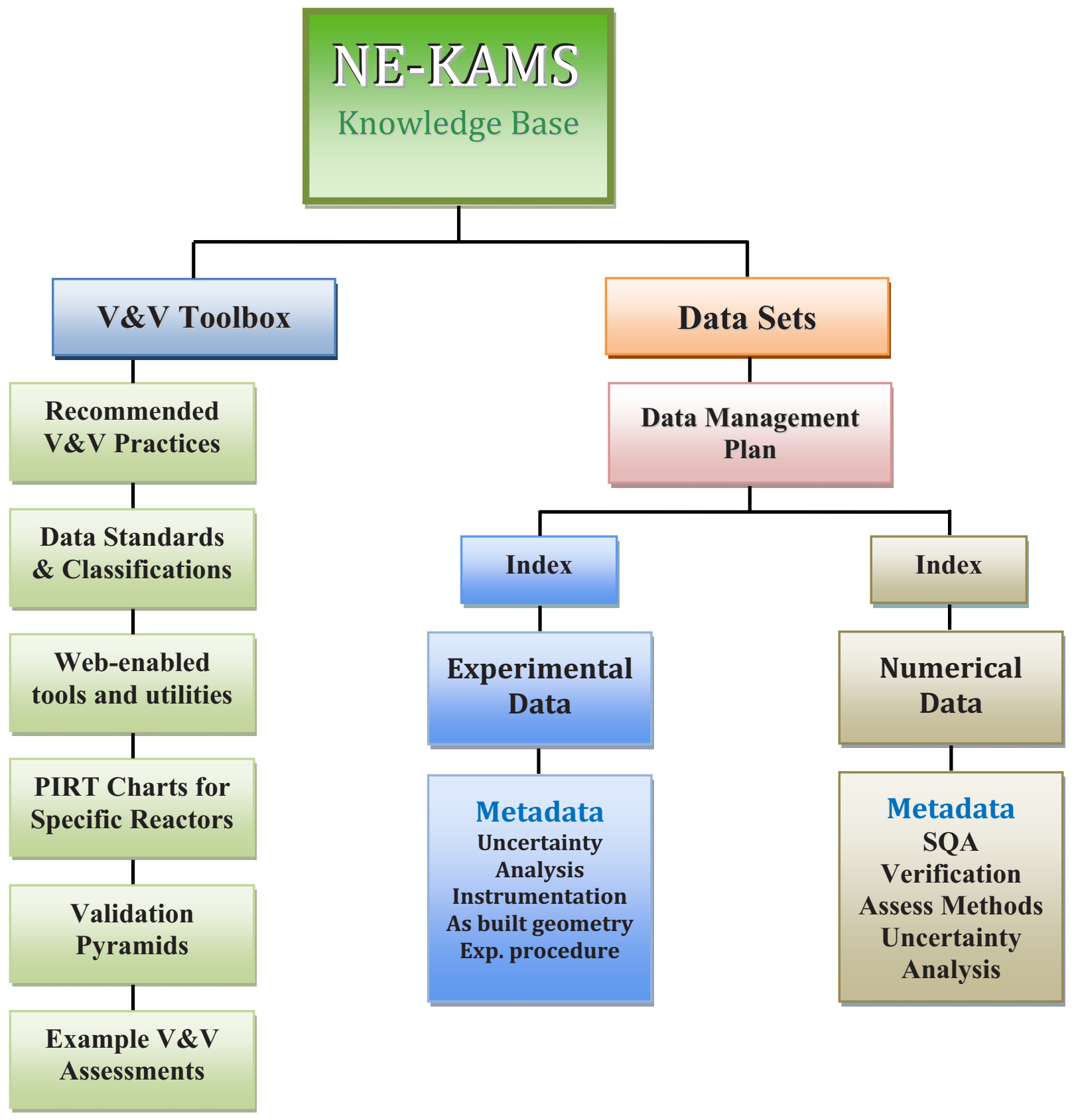

Figure 1. NE-KAMS Knowledge Base Elements 


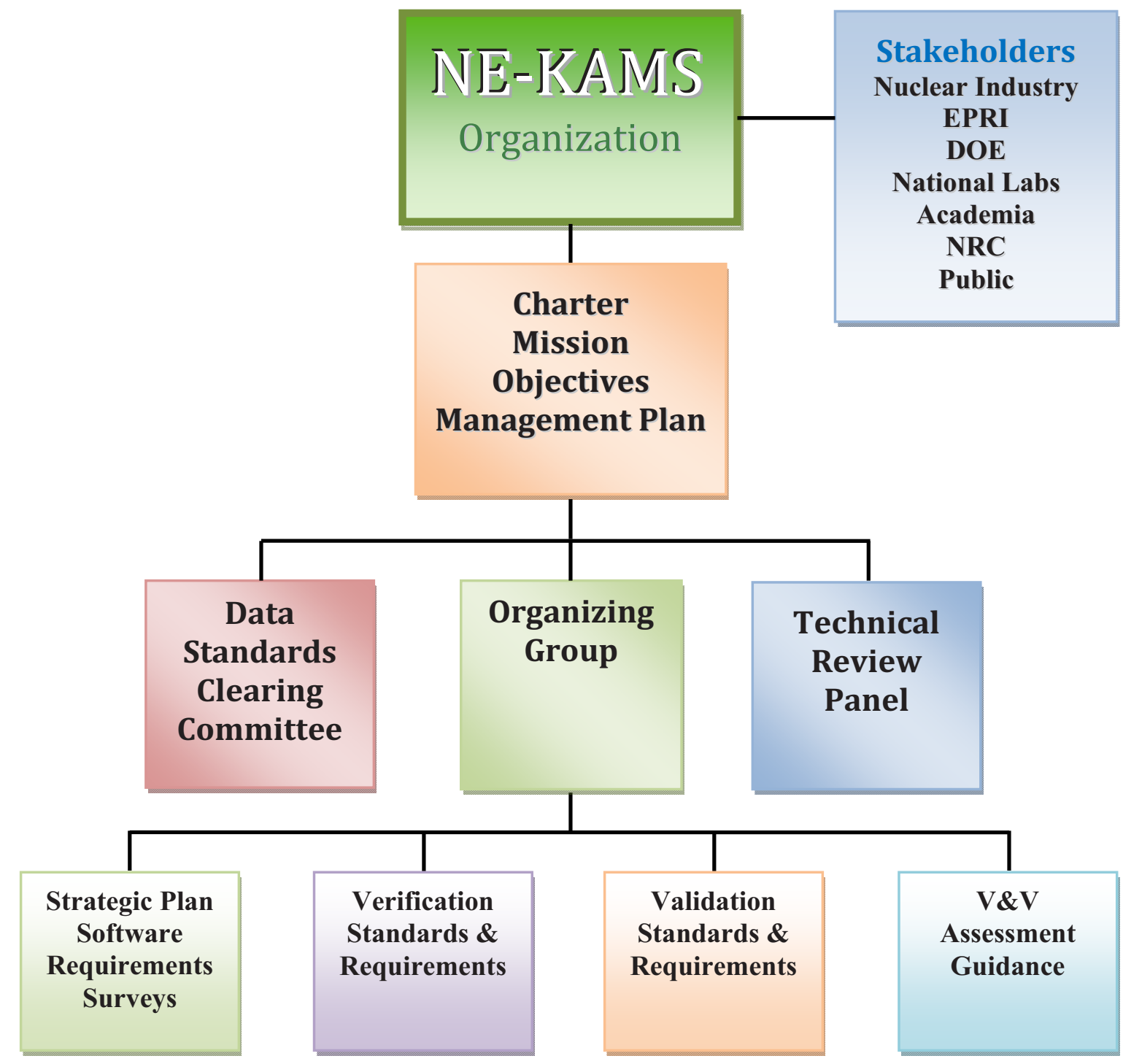

Figure 2. NE-KAMS Organization

\section{Verification and Validation of CFD for Nuclear Reactor Applications}

The initial focus for the NE-KAMS effort will be on supporting the use of CFD for M\&S of nuclear reactor systems, components and processes. To that end, standards, requirements and best practices for CFD V\&V will be established in support of the application of CFD to nuclear reactor design and safety analysis. It is not intended to provide any enforcement of such standards for those who employ V\&V data obtained from the NE-KAMS CFD V\&V databases. This is the responsibility of the cognizant authority for the application of any CFD analysis for a particular purpose. The inclusion of desired standards, requirements and best practices will provide the CFD analyst with guidance, help and understanding of how to perform accurate CFD analyses and provide confidence in the CFD analysis results. Appendix B provides details of the NE-KAMS recommended approach for V\&V of CFD models and simulations as well as other 
processes that help the analyst to review and assess the quality of CFD calculations. It is understood that the standards and procedures are still under development and will be subject to change in the future whenever new findings or research provide enlightenment and/or new methods.

\subsection{Current Status of CFD V\&V}

The application of CFD methods to problems relating to nuclear reactor design and safety analysis is less well developed than the traditional reactor system and containment codes, e.g., RELAP5, TRACE, and MELCOR, but progress is rapidly accelerating. This need to use CFD arises because many traditional reactor system and containment codes are based on networks of $1-\mathrm{D}$ or $0-\mathrm{D}$ volumes. It is evident, however, that the flow in such components as the upper and lower plena, downcomer and core of a reactor pressure vessel is strongly three-dimensional. Natural circulation, mixing, and stratification in containments is also essentially 3-D in nature, and representing such complex flows by pseudo 1-D approximations may not just be oversimplified, but could even be misleading, resulting in erroneous judgments being made.

The traditional approaches to nuclear reactor design and safety analysis, using system codes, for example, take advantage of the very large database of mass, momentum, and energy exchange correlations that have been built into them. The correlations have been formulated from essentially 1-D separate-effects experiments, and their range of validity is well known and controlled internally within the numerical models. Notwithstanding the scaling issues which still need to be resolved, therein lies the trustworthiness of the numerical predictions of system codes. Analogous databases for 3-D flows are very sparse by comparison, and the issue of the trust and reliability of CFD codes for use in nuclear reactor design and safety analysis applications has to be addressed before CFD can be considered at a similar level.

The conventional CFD V\&V approaches in use today in the nuclear industry are, by and large, ad hoc and application-specific. This is not surprising since CFD is used mostly for case basis, specific needs assessments and, as a result, most CFD users are interested in "validating" the CFD analysis and results for their specific application at hand. Thus, if a validation assessment were to be performed, it is likely that the validation assessment would be performed at the top tiers of the validation hierarchy, i.e., complete system and subsystem cases tiers, using test data obtained from large-scale, application-specific flow tests or actual testing of engineered systems. Nonetheless, there are CFD V\&V and application databases that presently exist, mostly outside of the nuclear domain, such as the European Research Community on Flow, Turbulence and Combustion (ERCOFTAC), European QNET-CFD, and NPARC Alliance databases. These CFD V\&V and application databases are essentially repositories for V\&V cases, CFD analysis results and related documents. There are no standards, requirements or best practices guidance for CFD $\mathrm{V} \& \mathrm{~V}$ associated with these $\mathrm{V} \& \mathrm{~V}$ data and application databases. There is also no process of quality assurance, such as review and approval procedure for entries into the database, associated with these databases. In fact, it can be stated that there is a little or no organizational control on the use of these databases. How one uses these databases is, by and large, up to the user. It is noted that over time, most of these CFD V\&V and application databases have either become defunct or fallen out of date due to lack of use and interest. 


\subsection{NE-KAMS CFD V\&V Approach}

The successful use of CFD for fluid dynamics analysis in the nuclear reactor design and safety analysis environments requires the attainment of acceptable levels of credibility of CFD simulations. The credibility of CFD simulations is, in turn, attained by quantifying acceptable levels of error and uncertainty. The primary means by which one can estimate and quantify levels of error and uncertainty and establish confidence in computational simulations are V\&V. $\mathrm{V} \& \mathrm{~V}$ bring an accepted system and structure to the process of evaluating the confidence to be placed in predictions from a given computational model, for a stated application. Therefore, to enable predictive CFD capability for use in nuclear reactor design and safety analysis requires the development and implementation of a focused, systematic program for CFD V\&V.

Such a focused approach for CFD V\&V must include the following principles:

- A V\&V program that supports computational tools in the establishment of a suitably inclusive validation domain;

- Dedicated CFD validation experiments that are conducted to obtain high-fidelity, highresolution flow field data from very well characterized experiments. This involves decomposition of fluid flow problems into unit and separate-effects physics employing PIRT charts and validation pyramids;

- Characterization and quantification of uncertainty in both the CFD simulation and experimental data to enable a predictive CFD capability that is based on best-estimate plus uncertainty approaches;

- A V\&V program that provides a knowledge base required to develop CFD best practices and experiment design guidelines;

- A V\&V program that is thoroughly and systematically documented and structured to support information and data management systems.

The NE-KAMS recommended approach for CFD V\&V emphasizes a bottom up view of accuracy assessments. In particular, $V \& V$ assessments of fundamental, unit and separate-effects physics, flow configurations are performed to develop the large body of knowledge required to implement best practices and design guidelines for managing uncertainty and improving reliability of CFD analysis and simulations. In this approach, the flow field data obtained from validation experiments require a higher level of fidelity, resolution and documentation, including a complete and thorough description of the boundary and initial conditions driving the flows, the as-tested geometry of the validation experiment, a detailed description of the measurement instruments used and measurement locations, and an appropriate uncertainty analysis of the experimental data. Datasets which meet these standards are termed validation-level datasets.

It is suggested that the NE-KAMS CFD V\&V approach outlined herein should create, over time, an extensive database of validation-level data that will be applicable to a wide variety of engineering applications. The knowledge gained from CFD V\&V assessments of validationlevel data will be used to assure the accuracy of the associated CFD predictions over a wide range of applications with a reduced amount of additional, confirmatory physical testing. In time, automated and standardized CFD methodology with associated best practices, design guidelines, and uncertainty quantification methods will provide a predictive capability in which sufficient confidence can be placed in CFD predictions that CFD analysis can replace large, semi-scale physical testing. Similarly, CFD analysis can be used with confidence in nuclear 
reactor safety analysis to bring real benefits in terms of better understanding and increased safety.

The development and implementation of NE-KAMS CFD V\&V database must be a joint effort of CFD code and methods developers, CFD code users and experimental groups that obtain the needed experimental data. A successful V\&V program for CFD will only be possible when the CFD development, CFD users and fluid dynamics experimental communities all work together in a coordinated effort.

\subsection{Precedents from Nuclear Reactor Physics}

Although developing and implementing a CFD V\&V database in support of CFD M\&S for nuclear reactor design and safety analysis applications will be a demanding task, there are precedents in the nuclear reactor physics community where similar efforts were conducted successfully. In the early years, the nuclear reactor physics community was in a similar situation as the CFD community is today with respect to CFD V\&V in that there were no accepted standards and formats for nuclear data. Each organization had to develop and maintain its own set of standards and formats for nuclear data which supported its own suite of nuclear reactor physics analysis and applications.

Collaborative efforts within the nuclear reactor physics community have since led to the establishment of accepted standards and formats for nuclear data which, in turn, have allowed the nuclear reactor physics community to leverage and take advantage of worldwide resources and expertise in nuclear reactor physics. Such collaborative efforts in nuclear reactor physics include:

- Cross Section Evaluation Working Group (CSEWG)

- International Criticality Safety Benchmark Project (ICSBEP)

- International Reactor Physics Experiment Evaluation Project (IRPhEP)

\section{NE-KAMS Implementation Plan}

This section provides the implementation strategy for NE-KAMS and describes anticipated activities for the future based on the premise that sufficient funding will be forthcoming.

\subsection{Implementation Strategy}

Initially the NE-KAMS effort will work on the implementation of all of the primary elements of the knowledge base: (a) the standards, requirements and best practices for V\&V and UQ assessments, (b) the quality standards for experimental and numerical benchmark data, (c) quality-assessed, web-accessible experimental and numerical benchmark databases, (d) a searchable knowledge base of information, documents, and data on V\&V and UQ, and (e) focused $\mathrm{V} \& \mathrm{~V}$ case studies in support of the nuclear industry. A graded approach will be followed in which preliminary but useful progress will be made for each element. With time, each element will be improved, revised and expanded as the development processes evolve and lessons learned are incorporated. This approach will also provide useful information and resources early on in the development of the knowledge base, especially for the DOE nuclear energy M\&S programs. 
The initial focus for the NE-KAMS effort will be on supporting the use of CFD for M\&S of nuclear reactor systems, components and processes. CFD validation datasets, both existing and legacy experimental data, will be sought first for inclusion in the NE-KAMS validation database. The initial standards, requirements and procedures for validation data evaluation and classification will be specific to CFD, but are expected to be generalizable. It is expected that the NE-KAMS CFD validation datasets will be composed of two types of experimental data:

- Single-phase flow experimental data from CFD validation experiments;

- Single-phase and heated flow experimental data from separate-effects $\mathrm{T} / \mathrm{H}$ and reactor systems validation experiments.

In addition, CFD V\&V assessments of a select set of existing and legacy experimental data will be performed to assess the uncertainty and applicability of $\mathrm{T} / \mathrm{H}$ analysis models and methods currently being used for nuclear reactor design and reactor safety analysis applications. Through these V\&V case studies, it is expected that guidelines and recommended practices for reducing uncertainty and improving applicability of $\mathrm{T} / \mathrm{H}$ analysis can be developed in support of $\mathrm{T} / \mathrm{H}$ analyses being performed in the nuclear industry. This industry-focused effort will evaluate legacy experimental data that is in common use for $\mathrm{T} / \mathrm{H}$ analysis, including experimental data for

- Wall friction

- Heat transfer

- Pressure loss coefficients

- Thermal and species mixing

- Natural convection and buoyant flows

- Multi-phase flows

This NE-KAMS effort will also consider and evaluate Code Scaling, Applicability, and Uncertainty (CSAU) methodology for CFD analysis, especially in the context of assessing the applicability of PIRT charts and validation pyramids.

Once NE-KAMS has reached a sufficient level of functionality for CFD and T/H analysis, the same graded approach will be applied to develop and implement other databases that are pertinent to nuclear reactor design and safety analysis, such as the materials and fuel system performance databases.

\subsection{Implementation Activities}

The specific tasks of the implementation plan for the development and establishment of NEKAMS are listed by fiscal year. It should be noted that these tasks are dependent on securing sufficient funding to pursue these tasks.

\section{Fiscal Year 2011 Activities}

- Establish a working group of professionals from the national labs and academia to define organize and develop the context for NE-KAMS; invite the NRC to observe and add commentary. Hold frequent planning meetings as deemed appropriate. Create sub-groups to address the individual elements of NE-KAMS and begin the development of these 
elements. Appendix A provides a list of working group members and the sub-groups that will address particular elements.

- Develop the standards, requirements and best practices for CFD V\&V, including a preliminary set of standards and classifications for validation experimental data.

- Establish software requirements for web-enabled information and data management infrastructure that will be needed to host the NE-KAMS database and its elements.

- Establish a Technical Review Panel.

- Seek out potential funding sources for 2012, in addition to the funding received from NEAMS and CASL.

- Identify and investigate existing CFD V\&V databases and best practice guidelines.

- Collect existing documented practices and procedures for CFD analysis from any and all sources.

- The activities for FY 2011 will result in several NE-KAMS planning documents including this strategic plan, a CFD V\&V data standards and requirements document, and a high level requirements document for the NE-KAMS databases and attendant information and data management system.

\section{Ongoing Activities for the foreseeable future}

- Maintain and consult with the Technical Review/Advisory Panel.

- Seek out potential funding sources for NE-KAMS.

- Develop and review standards and classifications for qualifying experimental data.

- Identify and investigate CFD V\&V databases.

- Develop and review standards, requirements and best practices for CFD V\&V and UQ.

- Collect new and legacy validation data and metadata for evaluation and classification and inclusion in NE-KAMS databases.

- Collect high fidelity numerical benchmark data in support of computational methods development.

In addition to the ongoing activities, specific tasks are listed for future years:

\section{Fiscal Year 2012 Activities}

- Assess existing software for the various elements of NE-KAMS, including website portal, search engines, data repository management, and data post-processing.

- Set up an initial website, not yet generally available, and begin testing the website software.

- Obtain experimental datasets and related metadata and use them to begin to establish the procedures for collecting, storing and classifying the data and making it accessible on the website.

- Obtain or develop validation pyramids and PIRT charts for reactors/systems related to CASL, NEAMS, LWRS, SMR and NGNP. 
- Obtain code verification benchmark data and related metadata to begin to establish the procedures for collecting, storing and classifying the data and making it accessible on the website.

- Conduct case studies to develop guidelines and recommended practices for using existing and legacy experimental data which will focus on reducing the uncertainty and improving the applicability of $\mathrm{T} / \mathrm{H}$ analyses currently being performed in industry.

- Fully establish the Data Standards Clearing Committee.

\section{Fiscal Year 2013 Activities}

- Incorporate systems software infrastructure determined to be appropriate for use with the NE-KAMS knowledge base.

- Obtain dedicated information and data management system software and hardware and attendant infrastructure, including facility, for the NE-KAMS knowledge base.

- Increase the NE-KAMS staff in preparation for the operational deployment of the NEKAMS knowledge base, including fully operation web portal and attendant information and database management system.

- Implement fully vetted procedures for data collection, processing, storage and maintenance.

\section{Fiscal Year 2014 Activities}

- Implement fully functional and operational systems software and hardware infrastructure for the NE-KAMS knowledge base.

- Add web-enabled tools and utilities for $\mathrm{V} \& \mathrm{~V}$ activities, data access and processing and physics discovery.

- Implement the fully vetted set of standards and procedures for evaluating and classifying validation experiment data.

- Implement the fully vetted standards, requirements and best practices for CFD V\&V of computational models and methods.

\section{Fiscal Year 2015 Activities}

- With many of the elements and procedures established for the CFD portion of the knowledge base, begin to develop and implement materials and fuel system performance databases.

- Add subject area experts to the Technical Advisory Panel and Data Standards Clearing Committee for materials and fuel system performance.

- Begin to establish standards for evaluating and classifying materials and fuel system performance data.

- Begin to establish standards, requirements and best practices for $\mathrm{V} \& \mathrm{~V}$ applied to materials and fuel system performance.

- Begin to collect data for materials and fuel system performance databases. 


\section{Out Years}

In the out years, the NE-KAMS knowledge base will continue to grow and include more V\&V benchmark data and associated metadata. The V\&V and UQ standards, requirements, procedures and best practices as well as related web-enabled tools and utilities will be subject to improvement and upgrading. Panels and committees will need to receive new members with expertise in the new technical areas added to NE-KAMS. Systems hardware and software infrastructure for the NE-KAMS knowledge base will need to be increased and upgraded to new technologies, as needed.

\section{NE-KAMS Implementation and Operations}

The NE-KAMS knowledge base will deploy state-of-the-art technology in information and data management systems and web services, leveraging the latest advances in high performance computing and web/internet technologies to maximize the benefit to its users in industry, academia and government. This section describes how the NE-KAMS knowledge base will be implemented and operated along with the attendant implementation challenges and issues as well as some of the benefits that will be derived from the NE-KAMS knowledge base once it is implemented.

\subsection{NE-KAMS Standards, Databases and Infrastructure}

The NE-KAMS knowledge base will be developed, implemented, and deployed for use in stages and will grow and evolve over time. In particular, the primary elements of the knowledge base along with the attendant hardware, software and facility infrastructure will be developed and implemented in stages as funding and available resources allow. Similarly, the knowledge base will be deployed for use in stages where select parts of the knowledge base, e.g., standards, requirements and best practices for $\mathrm{V} \& \mathrm{~V}$ and $\mathrm{UQ}$ of computational models and simulations, will become available for use when ready.

The following elements of the NE-KAMS knowledge base will be developed, implemented and deployed:

- Standards, requirements and best practices for performing V\&V and UQ assessments of computational models and simulations for nuclear reactor design and safety analysis will be developed and implemented. Initially the NE-KAMS effort will focus on developing and implementing V\&V standards, requirements and best practices. This effort will build from and leverage previous efforts in V\&V standards and guides of the American Institute of Aeronautics and Astronautics (AIAA) and the American Society of Mechanical Engineers (ASME). In addition, NE-KAMS will coordinate all future work in $\mathrm{V} \& \mathrm{~V}$ standards, guides and practices with these organizations and with technical organizations in the nuclear energy sciences and engineering, such as the American Nuclear Society (ANS) and the Organization for Economic Cooperation and Development/Nuclear Energy Agency (OECD/NEA). It is envisioned that these standards, requirements and best practices for $\mathrm{V} \& \mathrm{~V}$ developed by the NE-KAMS effort should become the accepted standards, requirements and best practices for $\mathrm{V} \& \mathrm{~V}$ of computational models and simulations for the various nuclear energy sciences and engineering communities. 
- Standards and procedures for the evaluation and classification of experimental and numerical benchmark data for use in $\mathrm{V} \& \mathrm{~V}$ assessments of computational models and simulations will be developed and implemented. These standards and procedures will additionally provide clear guidelines on what is required to generate high-quality $\mathrm{V} \& \mathrm{~V}$ benchmark data by highlighting the importance of several key quality classification categories. These standards and procedures can also be used to assess the pedigree of legacy experimental data used to develop physical models and correlations, which, in turn, will be useful in quantifying the uncertainty and assessing the applicability of $\mathrm{T} / \mathrm{H}$ analysis models and methods currently being used in the nuclear industry. This NEKAMS effort is unique in that currently no accepted standards or procedures exist for assessing the quality of experimental and numerical benchmark data used in $\mathrm{V} \& \mathrm{~V}$ assessments. It is envisioned that these standards and procedures developed by the NEKAMS effort can become the accepted standards and procedures for assessing and classifying experimental validation and code verification benchmark data for use in V\&V of computational models and simulations for the various nuclear energy sciences and engineering communities.

- Quality-assessed, web-accessible databases for nuclear energy related experimental and code verification benchmark data and metadata that can be used in V\&V assessments will be developed, implemented and deployed. It is expected that over time, an extensive database of $\mathrm{V} \& \mathrm{~V}$ benchmark cases will be developed that are applicable to a wide variety of applications in nuclear energy sciences and engineering. Most V\&V and applications databases, such as the ERCOFTAC, QNET-CFD, NPARC Alliance, and OECD/NEA databases, serve only as repositories for $\mathrm{V} \& \mathrm{~V}$ cases and related documents. The NEKAMS V\&V benchmark databases will be supported by and used in conjunction with the NE-KAMS V\&V standards, requirements, and best practices, providing a unique resource and capability for $\mathrm{V} \& \mathrm{~V}$ and quality assurance of computational models and simulations. It is expected that the NE-KAMS V\&V benchmark databases will become a valuable resource for the nuclear industry, academia, national laboratories and the U.S. NRC.

- A knowledge base of searchable information, documents and data related to $\mathrm{V} \& \mathrm{~V}$ and UQ of computational models and simulations will be developed, implemented and deployed. The NE-KAMS knowledge base will include such items as examples of credible V\&V and UQ assessments, validation pyramids and PIRT charts for applicable nuclear reactor designs and systems, and reference documents, technical reports and journal papers on $\mathrm{V} \& \mathrm{~V}$ and $\mathrm{UQ}$. It is envisioned that over time a large amount of information and data on V\&V and UQ will be collected, processed and formatted and made available to NE-KAMS knowledge base users as a searchable knowledge base. All the information, documents and data will not reside in the NE-KAMS knowledge base. Appropriate links and connections will be provided to other sources of information, documents and data, including other data and knowledge bases, as needed. It is thus envisioned that the NE-KAMS knowledge base will become a valuable resource of cohesive reference information and data for the nuclear industry, academia, national laboratories and the U.S. NRC.

The NE-KAMS knowledge base will maximize the benefits to its users by providing the means to access rapidly and coherently the required information and data and efficiently and effectively utilize them in support of $\mathrm{V} \& \mathrm{~V}$ and UQ assessments and methods development. To that end, NE-KAMS will deploy state-of-the-art technology in information management systems and web 
services to leverage high performance computing and web technologies. The computing infrastructure will include knowledge base systems software, web-based user interface systems, high performance computing and storage systems, and high performance networks. Webenabled applications, tools and utilities for V\&V and UQ activities, data access and processing, and information and data searches will be developed, implemented and deployed.

The NE-KAMS approach to computing will be unique in that the users can interact with the knowledge base and perform tasks that would have to be done offline with conventional databases, which typically only allow for downloading of information and data. It is envisioned that with the full deployment of the NE-KAMS knowledge base, much, if not all, of the V\&V and UQ tasks of the user will be performed in the NE-KAMS knowledge base. It is thus argued that the NE-KAMS knowledge base, when fully implemented and deployed, will bring the power of high performance computing to the masses in that there will no longer be technical or financial barriers for accessing the powers of high performance computing for NE-KAMS knowledge base users.

As mentioned above, it is expected that the NE-KAMS knowledge base will have links to other data and knowledge bases, especially those of the DOE, such as the Office of Scientific and Technical Information (OSTI), and, as such, will serve as an access portal for a much larger and more extensive body of knowledge to it users. The NE-KAMS knowledge base will be implemented and operated with the required security infrastructure and protocols to accommodate both proprietary and public data and information.

The realization of the described vision for NE-KAMS knowledge base will require a focused effort with sustained funding and dedicated staff and resources for several years. To that end, it should be recognized that the initial implementation of the NE-KAMS knowledge base will be technically and organizationally complex, as well as costly, and the long-term success of the NEKAMS knowledge base requires a sound starting point, with broad consensus from all the interested parties about goals, use, access and funding over the long term.

Additional details on the NE-KAMS knowledge base implementation will be provided in the two NE-KAMS planning documents:

- $\quad$ NE-KAMS Knowledge Base High Level Requirements

- NE-KAMS Knowledge Base Code Verification and Validation Data Standards and Requirements

\subsection{NE-KAMS Challenges and Issues}

There are several challenges and issues associated with NE-KAMS that need to be addressed:

- Populating the database with high quality CFD V\&V data and assessments will require a wide-ranging effort that cuts across major communities of applied mathematics, model building, experiment, computation, engineering applications and business decision making;

- Putting this kind of collaborative effort together hinges on a careful plan that takes a long-term view for the knowledge base; 
- Because of the wide variety of thermo-fluid conditions in nuclear reactor thermal hydraulics, a large number of small scale, unit physics or separate-effects experiments will be required to develop the knowledge base and technical underpinning for $\mathrm{V} \& \mathrm{~V}$ processes at the base of the validation pyramid;

- The resources needed to collect and process large, petabyte-size data sets and the infrastructure required to store and manage them are not readily available;

- Proprietary information and data can be difficult to obtain and share, and in general cannot be shared with non-US citizens.

\subsection{NE-KAMS Benefits}

Several benefits will be realized with the implementation of the NE-KAMS knowledge base.

- V\&V Data Sharing - Quality-assessed, web-accessible databases of high-quality experimental and numerical benchmark data that can be shared will be very useful for the nuclear energy sciences and engineering communities. Such databases will not only provide valuable assistance and convenience to researchers, analysts and regulators, but also provide confidence in the quality of the benchmark data used in V\&V assessments.

- Expertise - The V\&V and UQ expertise of experimentalists, methods developers, and computational analysts as provided in the NE-KAMS standards, best practices, V\&V benchmark databases and knowledge base, will be very helpful to researchers and analysts in industry, government, and academia performing V\&V and UQ assessments.

- $\boldsymbol{V} \boldsymbol{\&} \boldsymbol{V}$ Information - The V\&V related information in NE-KAMS, especially the PIRT charts and validation pyramids, will help standardize and centralize the V\&V information and make it easier for researchers and analysts to access the required information and perform their $\mathrm{V} \& \mathrm{~V}$ assessments.

- Preservation of Data - NE-KAMS will provide an ongoing repository for data to ensure that it is not lost to posterity. This will prevent expenditures to reproduce the data and even just the effort that would be required to dig out legacy data from wherever it would otherwise have been stored. NE-KAMS will also over time update its information and data management infrastructure, i.e., hardware, software and facility, so that it will always be readily accessible to its users.

- Culture of Quality - The widespread use of NE-KAMS will create an incentive to produce "benchmark-quality" products and encourage researchers, developers and analysts to perform uncertainty quantification and follow recommended best practices. Also, it will motivate experimentalists to produce benchmark data that can be qualified for inclusion in NE-KAMS databases.

\section{Concluding Remarks}

Because the nuclear energy community is geographically dispersed and fragmented, effectively communicating and collaborating with one another is difficult and unreliable. However, this situation can be greatly improved by making important $V \& V$ data and related metadata readily available to the nuclear energy sciences and engineering communities through a trusted knowledge base for technical exchange and collaboration. Thus, we recognize the strategic importance of implementing NE-KAMS and ensuring that it aligns with the business needs of the 
nuclear energy sciences and engineering communities. More precisely, the business case for establishing NE-KAMS is simple: Without a concerted effort to preserve, evaluate, document and disseminate existing nuclear reactor systems design and safety analysis models and data, over time, they will be irretrievably lost. The money and resources invested to create them will be wasted and future generations will have to duplicate the R\&D work to regenerate these models and data if nuclear energy is to be a factor in our energy future.

\section{Reference Materials}

Quality Staff. Guidance on Environmental Data Verification and Validation, EPA QA/G-8, November 2002.

Larzelere, A. R. Advanced Modeling and Simulation Facilities Requirements, presentation to the Office of Nuclear Energy, Fuel Cycle Management (NE-5), 2009.

Pasamehmetoglu, K. O. A Science-Based Approach to Nuclear Fuel Cycle Development, Formal Report, Idaho National Laboratory, April 2009.

Oberkampf, W.L., Roy, C. J. Verification and Validation in Scientific Computing, Cambridge University Press, Cambridge, 2010.

Lee, H. B. and Bauer, R. C. Predictive Computational Fluid Dynamics Development and Its Verification and Validation: An Overview, paper FEDSM2009-78147, Proceedings of the ASME 2009 Fluids Engineering Division Summer Meeting, Vail, Colorado, Aug. 2-6, 2009.

Dalkir, K. Knowledge Management in Theory and Practice, Elsevier Inc., 2005. 


\section{APPENDIX A}

\section{NE-KAMS WORKING GROUP AND SUB-GROUPS}

\begin{tabular}{|c|c|}
\hline king C & cs $\mathrm{Ob}$ \\
\hline James Peltz & DOE, Nuclear Energy \\
\hline Kerry Webb & DOE, Nuclear Energy \\
\hline Chris Boyd & US NRC (observer) \\
\hline Hope Forsmann & Idaho National Laboratory \\
\hline James Fort & Pacific Northwest National Lab. \\
\hline Rich Johnson & Idaho National Laboratory \\
\hline Hyung Lee & Bettis Laboratory \\
\hline Robin McCollum & Bettis Laboratory \\
\hline Kimberlyn Mousseau & Idaho National Laboratory \\
\hline Vince Mousseau & Sandia National Laboratories \\
\hline William Oberkampf & Oberkampf Consulting \\
\hline Dave Pointer & Argonne National Laboratory \\
\hline Bill Rider & Sandia National Laboratories \\
\hline Bart Smith & Utah State University \\
\hline James Stewart & Sandia National Laboratories \\
\hline Laura Swiler & Sandia National Laboratories \\
\hline Tim Trucano & Sandia National Laboratories \\
\hline Greg Weirs & Sandia National Laboratories \\
\hline Tech & $I$ and Affil \\
\hline Nam Dinh & Idaho National Laboratory \\
\hline Thomas Downar & University of Michigan \\
\hline James Fort & Pacific Northwest National Lab \\
\hline Annalisa Manera & University of Michigan \\
\hline Robin McCollum & Bettis Laboratory \\
\hline William Oberkampf & Oberkampf Consulting \\
\hline Ronaldo Szilard & Idaho National Laboratory \\
\hline
\end{tabular}

james.peltz@nuclear.energy.gov kerry.webb@nuclear.energy.gov christopher.boyd@nrc.gov

hope.forsmann@inl.gov james.fort@pnl.gov rich.johnson@inl.gov hyung.lee@unnpp.gov Robin.Mccollum@unnpp.gov kimberlyn.mousseau@inl.gov vamouss@sandia.gov wloconsulting@gmail.com dave.pointer@anl.gov wjrider@sandia.gov bsmith@engineering.usu.edu jrstewa@sandia.gov lpswile@sandia.gov tgtruca@sandia.gov vgweirs@sandia.gov

nam.dinh@inl.gov downar@umich.edu james.fort@pnl.gov manera@umich.edu robin.mccollum@unnpp.gov wloconsulting@gmail.com ronaldo.szilard@inl.gov

Sub-group committee assignments (Feb. 3, 2011)

1. Technical Panel

- Lead - Kim Mousseau

2. Strategic Plan

- Lead - Kim Mousseau

- Hyung Lee

- Rich Johnson

- Dave Pointer

- Kerry Webb

\section{Survey of what currently exists}

- Lead-Hyung Lee 


\section{Data V\&V}

Verification Standards and Requirements

- Lead - Bill Rider

- Greg Weirs

- William Oberkampf

- Jim Fort

Validation Standards and Requirements

- Lead - Bart Smith

- Greg Weirs

- Hyung Lee

- William Oberkampf

- Jim Fort

- Nam Dinh

Taxonomy

- Lead-Greg Weirs

- Bill Rider

- William Oberkampf

- Bart Smith

$V \& V$ Assessment Guidance

- Lead-Hyung Lee

- Rich Johnson

- William Oberkampf

- Jim Fort

- Greg Weirs

\section{Software Requirements Document}

- Lead - Kim Mousseau

- Rich Johnson

- Hyung Lee

- Dave Pointer

- Kerry Webb

6. Analysis of What Currently Exists

- Lead - Hyung Lee

7. Review and Participation

- Lead - Jim Fort

- Tim Trucano

\section{Sandia Liaison}

- Greg Weirs 


\section{APPENDIX B}

\section{CFD V\&V DATABASE}

\section{Verification and Validation of Computational Fluid Dynamics Models and Simulations for Nuclear Reactor Applications}

The complexity of modern engineering systems, as well as the cost and difficulty associated with experimentally testing system and subsystem design makes the use of modeling and simulation (M\&S) a desirable tool for design and development. The predictive ability of numerical simulation, such as computational fluid dynamics (CFD) and computational structural mechanics (CSM) has matured significantly. However, for M\&S to be used with confidence in design and development, quantitative measures of uncertainty must be available. As such, methods for assessing computational uncertainty are one of the focused areas of research for the computational science and engineering community. In general, the approaches for dealing with computational uncertainty can be divided into model verification and validation (V\&V), i.e., code verification and model validation, and prediction uncertainty quantification (UQ), i.e., solution verification, uncertainty analysis, uncertainty propagation, and sensitivity analysis. The generally accepted definitions for these terms are given below:

\section{Model V\&V}

- Code verification - The process of gathering evidence that the mathematical model as implemented and its solution are correct.

- Model validation - The quantitative determination that the model is an accurate representation of the real world for its intended use.

\section{Prediction UQ}

- Solution verification - The estimation of discretization error.

- Uncertainty analysis - The identification and characterization of uncertainty sources.

- Uncertainty propagation - The propagation of input uncertainty into the system response quantities.

- Sensitivity analysis - The determination of the most important uncertainty contributors to system response quantities.

As a part of the NE-KAMS knowledge base for nuclear energy sciences and engineering, the current effort will develop and implement a CFD V\&V database. In support of the NE-KAMS CFD V\&V database, the NE-KAMS effort will develop standards, requirements and best practices for $\mathrm{V} \& \mathrm{~V}$ that will be used in $\mathrm{V} \& \mathrm{~V}$ assessments of CFD models and simulations used in nuclear reactor systems design and safety analysis. As an adjunct to its focused V\&V development activities and attendant V\&V case studies, the NE-KAMS effort will support the use of CFD in the nuclear industry by developing guidelines and recommended practices for applying CFD to nuclear reactor design and safety analysis. When completed, the NE-KAMS CFD V\&V database will include experimental and high fidelity code verification benchmark datasets that can be used in V\&V and UQ assessments and CFD analysis methods development. In addition, the NE-KAMS CFD V\&V database will include a suite of web-enabled utilities and 
tools for $\mathrm{V} \& \mathrm{~V}$ and UQ procedures as a part of the general quality assurance process for the use of CFD analysis in nuclear systems design and safety analysis.

\section{NE-KAMS CFD V\&V Approach}

Figure 1 [1] indicates that the flow fields for a practical thermal hydraulic design or application problem are in reality composed of a hierarchy of "physical interactions." Any application or problem can be decomposed into its unit or separate-effects physics using a procedure analogous to PIRT (Phenomenon Identification and Ranking Table). This, in turn, allows one to develop a better and more comprehensive understanding of the underlying fluid flow physics affecting the specific problem at hand by assessing the physics models at its unit and separate-effects physics levels of the validation hierarchy. To be useful for design and development, however, the knowledge developed from performing these CFD V\&V assessments has to be provided to the users as a structured predictive capability that can be used in design related CFD analyses and assessments over a wide application domain.

The basis of the NE-KAMS approach is to provide a firm underpinning of the underlying unit and separate-effects physics for each "flow path" of interest to a designer or analyst in a structured way. This approach is denoted as the "bottom-up approach to validation" and the validation hierarchy concept is identified as the validation pyramid to denote the broad base of unit and separate-effects physics validation assessments needed to demonstrate the predictive capability of CFD [2].

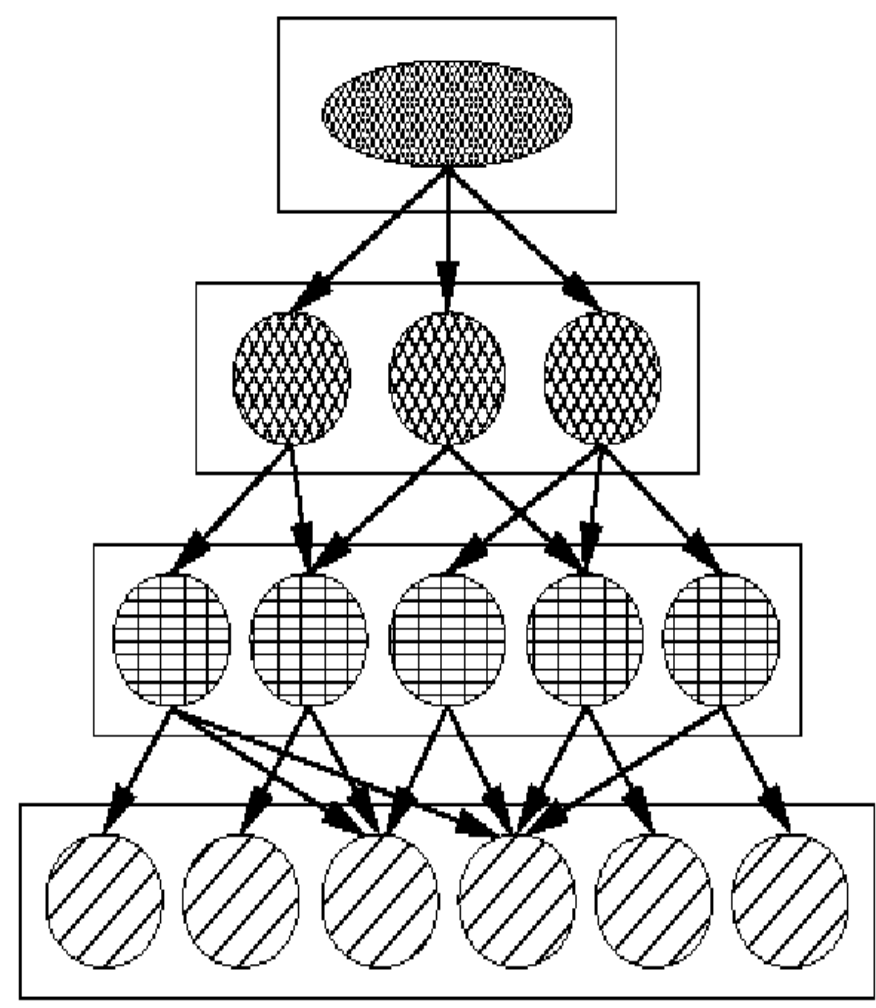

Complete System

Subsystem Cases

Benchmark Cases

Unit Problems

Figure 1 - Validation Hierarchy (From the AIAA Guide, 1998) 
Motivation for the Bottom-Up Approach for CFD Verification and Validation

The enormous potential of CFD for its use in the nuclear industry is predicated on the fact that CFD is a generally applicable tool. As such, CFD can be applied to simulate a diverse range of physics, conditions and geometries of interest to the nuclear industry. Thus, the objective of the NE-KAMS effort is the development of a systematic approach for CFD V\&V which is not only generic, but also flexible enough to demonstrate an acceptable level of credibility for CFD analysis and simulations for a wide range of fluid flow conditions and applications. It is this need to come up with an approach that supports a generally applicable tool that led us to settle on the "bottom-up" approach to validation for use in the NE-KAMS CFD V\&V database. The term "bottom-up" is used to denote the fact that CFD V\&V activities are focused at the lower tiers or the base of the validation pyramid to generate the large number of generally applicable validation datasets required to support the large number of applications at the top or complete system tier of the validation pyramid. It is noted that NE-KAMS approach does not preclude "top-down" approach for validation. In fact, the whole point of leveraging validation hierarchy (or PIRT combined with validation pyramid) in $\mathrm{V} \& \mathrm{~V}$ activities is to be able to do both bottom-up and topdown validation assessments.

In the NE-KAMS approach, by focusing the CFD V\&V activities on the lower tiers or the base of the validation pyramid, validation assessments become applicable to more than one application. In fact, it can be argued that for most CFD applications, when the complete systems of the applications are decomposed to their lower constituent tiers, many of the unit and separateeffects physics embodied in the unit problems and benchmark tiers would be applicable to most, if not all, of the complete systems at the top tier. As such, it can be stated that the NE-KAMS approach for CFD V\&V is physics-based, in contrast to the traditional approach which can be described as application-specific or application-centric. That is, the traditional approach is based on a "top-down" perspective of validation in which validation is performed for each specific thermal hydraulics $(\mathrm{T} / \mathrm{H})$ application at the top tier of the validation pyramid. The problem with the traditional approach, as practiced today, is not starting at the top and moving down, but, rather, starting at top and never doing anything else. The bottom-up approach is also more costeffective in that a validation dataset at the lower tiers of the validation pyramid could potentially support or be applicable to a number of applications at the top or complete system tier of the validation pyramid.

One can better understand this perspective of CFD V\&V when one examines the relationship of prediction to validation as shown in Figure 2 [3]. The "validation domain" in the figure suggests three features. First, in the validation domain we have high confidence that the relevant physics is understood and modeled at a level that is commensurate with the needs of the application. Second, this confidence has to be quantitatively demonstrated by satisfactory agreement between computations and experiments in the validation assessments for some range of applicable parameters in the computational model. And third, the boundary of the domain indicates that outside this region there is degradation in confidence in the quantitative predictive capability of the computational model. Stated differently, outside the validation domain the computational model is credible, but its quantitative capability has not been demonstrated. The "application domain" indicates the region where predictive capability is needed from the computational model for the application of interest. It is the goal of the NE-KAMS effort to maximize the coverage of the validation domain and the application domain for practical $\mathrm{T} / \mathrm{H}$ problems of interest to the nuclear industry. 
Figure 2a depicts the desired situation in engineering where there is a complete overlap of the validation domain with application domain and this is the desired goal of any $\mathrm{V} \& \mathrm{~V}$ effort. Figure $2 \mathrm{a}$ would be representative of a situation where a comprehensive set of CFD validation assessments sufficiently covers all the tiers of the validation pyramid. Figure $2 b$ depicts a situation where there is a significant overlap between the validation domain and the application domain. Figure $2 \mathrm{~b}$ could be representative of a situation where the set of CFD validation assessments covers some, but not all the tiers of the validation pyramid. Figure $2 \mathrm{~b}$ could be representative of a situation where the set of CFD validation assessments is limited to a specific tier of the validation pyramid. Figure $2 \mathrm{c}$ depicts the situation where there is no overlap between the validation domain and the application domain.

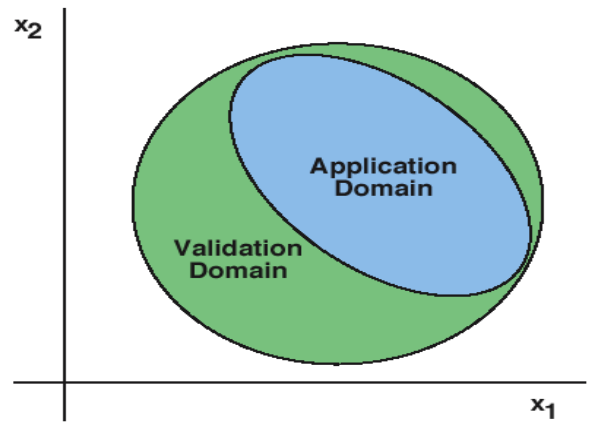

a) Complete Overlap

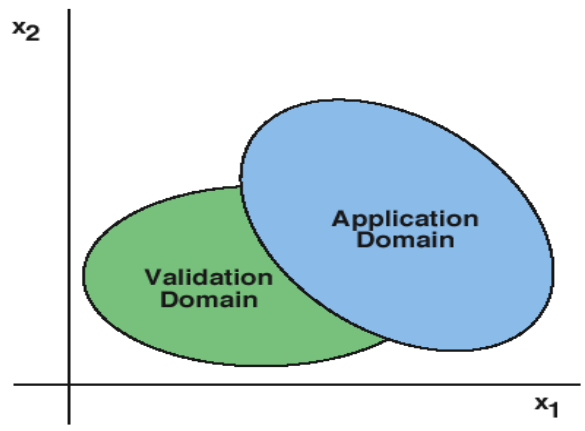

b) Partial Overlap

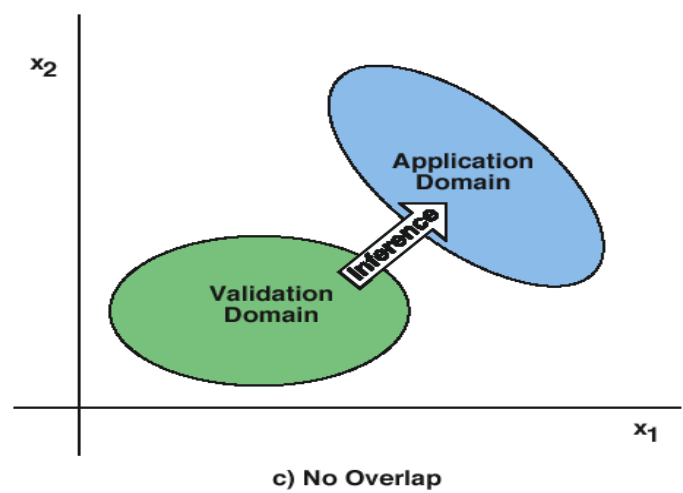

Figure 2 - Relationships of the Validation Domain to the Application Domain (From Oberkampf et al., 2002)

The V\&V of CFD simulations in the nuclear industry has been and continues to be viewed, by and large, as a top-down process in the validation pyramid, i.e., an application centric process. This is not surprising since in industry CFD is used mostly for case basis, specific needs assessments and, as a result, most CFD users are interested in "validating" the CFD analysis and results for their specific application at hand. Thus, if a validation assessment were to be performed, it is likely that the validation assessment would be performed at the top tiers of the validation pyramid, i.e., complete system and subsystem tiers, using test data obtained from large-scale, application-specific flow tests or actual testing of engineered systems. It is often impractical or impossible to acquire the detailed, local flow field data required for CFD validation assessments from these large-scale, application-specific tests due to cost, schedule, or test unit / test facility limitations. Instead, the data obtained from these tests are typically system level, integral or averaged measurements of flow field variables and related engineering data, 
e.g., lift, drag, pressure loss, mixing and heat transfer coefficients, which are more useful for assessing the performance of a specific design or engineered system. In addition, the amount of data obtained from these tests is often limited and data may include high levels of uncertainty associated with it. As such, CFD validation assessments performed only at the complete system and subsystem tiers of the validation pyramid are of limited value unless there is also a comprehensive set of underpinning validation assessments of unit and separate-effects physics flow cases. This is because validation assessments at the complete system and subsystem tiers often involve making comparisons with a limited amount of potentially highly uncertain test data.

It is suggested that the CFD V\&V approach outlined herein should create, over time, an extensive validation database that would be applicable to a wide variety of engineering applications. This validation database made up predominantly of physics-based, unit and separate-effects physics validation assessments can then serve as a vehicle for developing and extending a generally applicable knowledge base from which validation to higher levels of confidence for a given application can be performed, as needed. In fact, as the validation database gets well populated with these more detailed, physics-based validation assessments, it is expected that extending an existing set of validation assessments to a specific application will become more straightforward and comparatively fewer validation assessments will be needed. In time, validation assessments for new applications will begin to systematically use and leverage existing validation assessments in the validation database so that synergy will be maximized and duplication and redundancy will be minimized. Thus, over time, the validation domain will begin to approach the application domain for large classes of $\mathrm{T} / \mathrm{H}$ problems and analyses needed by the designers and developers.

The NE-KAMS approach has some other significant and practical advantages. First, because the underpinning validation assessments are performed predominantly at the base of the validation pyramid, one is dealing with simpler geometry and physics, which, in turn, makes the difficult tasks of a validation assessment more tractable. For instance, assessing and quantifying the uncertainty of computational results (grid convergence study and numerical error estimation) and experimental data (uncertainty analysis and propagation of uncertainty) are far easier tasks when one is dealing with the simpler geometries and flow physics at the lower tiers of the validation pyramid. Second, because in this approach one is performing validation assessments with highquality, high-resolution flow field data obtained from validation-level experiments, one is more likely to develop a better understanding of various physical phenomena embodied in the validation experiments. This better understanding of physical phenomena, in turn, can be used to develop knowledge-based procedures for applying CFD or aid in developing physical models. As such, in the NE-KAMS approach, the high-fidelity, high-resolution experimental data obtained from validation experiments can be used for other design related activities, such as physical modeling and methods development, in addition to validation assessments. Finally, the most important advantage of the NE-KAMS approach is that it can foster and promote collaboration among interested parties and organizations in expanding the validation range of CFD analysis to cover a larger application domain. In particular, because most of the flow geometries and unit or separate-effects physics at the base of the validation pyramid are not likely to be directly related to a proprietary application or design, interested parties are more likely to collaborate and share CFD V\&V resources and knowledge. It is in everyone's best interest to have access to a well populated CFD validation database made up of fundamental, unit and separate-effects physics validation assessments to draw upon to support their CFD V\&V efforts. 


\section{References}

1. AIAA Committee on Standards in CFD. Guide for Verification and Validation of Computational Fluid Dynamics, American Institute of Aeronautics and Astronautics, 1998.

2. Lee, H. B. and Bauer, R. C. Predictive Computational Fluid Dynamics Development and Its Verification and Validation: An Overview, paper FEDSM2009-78147, Proceedings of the ASME 2009 Fluids Engineering Division Summer Meeting, Vail, Colorado, Aug. 2-6, 2009.

3. Oberkampf, W. L. and Trucano, T. G. Verification and Validation in Computational Fluid Dynamics, SAND2001-0529, Sandia National Laboratories, 2002. 


\section{APPENDIX C}

\section{REACTOR SYSTEMS V\&V DATABASE}

\section{Verification and Validation of Reactor Systems Models and Simulations for Nuclear Reactor Safety Applications}

As a part of the NE-KAMS knowledge base for nuclear energy sciences and engineering, the current effort will develop and implement a verification and validation (V\&V) database that supports reactor systems codes and reactor safety analysis. This V\&V database will be an adjunct to the computational fluid dynamics (CFD) V\&V database, and, when implemented, will include both separate-effects and integral-effects experimental and numerical benchmark data that can be used in V\&V and UQ assessments of reactor systems models and simulations. The NE-KAMS effort, through its focused CFD V\&V development activities and attendant V\&V case studies, will aid in the development of guidelines or recommended practices aimed at reducing uncertainty and improving applicability of reactor systems codes and reactor safety analysis methods.

\section{NE-KAMS Reactor Systems V\&V Approach}

The objective of the NE-KAMS Reactor Systems V\&V database is to provide separate- and integral-effects experimental and numerical benchmark datasets that can be used in V\&V and UQ assessments of both existing and next generation reactor systems models and simulations. In the coming years, the NE-KAMS effort will work with the industry and academia in identifying and populating the NE-KAMS Reactor Systems V\&V database with separate- and integraleffects experimental and numerical benchmark data and related metadata for use in $\mathrm{V} \& \mathrm{~V}$ and UQ assessments. This effort is aimed at capturing and preserving existing and legacy thermal hydraulic $(\mathrm{T} / \mathrm{H})$ datasets that have been used in V\&V and UQ assessments of reactor systems codes and providing a working framework for obtaining and incorporating new $\mathrm{T} / \mathrm{H}$ data into the Reactor Systems V\&V benchmark database. This effort will leverage, as much as possible, the work that is being performed in the development and implementation of the NE-KAMS CFD $\mathrm{V} \& \mathrm{~V}$ benchmark database.

In addition, it is expected that the NE-KAMS effort will support the use of existing reactor system codes in industry by aiding in the development of guidelines or recommended practices aimed at reducing the uncertainty and improving the applicability of physical models and correlations used in reactor systems codes. In the near term, CFD V\&V assessments of a select set of existing and legacy experimental data will be performed to assess the uncertainty and applicability of $\mathrm{T} / \mathrm{H}$ analysis models and methods currently being used for nuclear reactor design and reactor safety analysis applications. This industry focused effort will evaluate legacy experimental data that is in common use for $\mathrm{T} / \mathrm{H}$ analysis, including experimental data for wall friction, heat transfer, pressure loss coefficients, thermal and species mixing, and natural convection and buoyant flows

In addition, it is judged that NE-KAMS Reactor Systems V\&V database, when implemented, can also be used in V\&V assessments of coupled CFD and reactor systems models and simulations. The issue is how does one verify and validate a coupled CFD and reactor systems model and 
simulation? What is clear is that it is not sufficient to verify and validate the reactor systems code and CFD models and simulations separately: the coupled model and simulation has to be verified and validated, and, thus, the validation process for a coupled CFD and reactor systems code model and simulation may have to involve integral-effects type of test data from reactor systems code benchmark cases.

In conjunction with these efforts, the NE-KAMS Reactor Systems V\&V database will focus on supporting the $\mathrm{V} \& \mathrm{~V}$ activities and processes for the use of next generation reactor systems code, R7 [1], in the Department of Energy (DOE) Office of Nuclear Energy sponsored Light Water Reactor Sustainability (LWRS) Program. The NE-KAMS effort will work closely with the LWRS Program to incorporate the NE-KAMS standards, requirements and best practices for $\mathrm{V} \& \mathrm{~V}$ and UQ into various LWRS V\&V and UQ activities and processes, especially those for the next generation reactor systems code R7. Similarly, the NE-KAMS effort will work with the LWRS Program to identify its V\&V and UQ needs and initiate the process to develop and implement the NE-KAMS Reactor Systems V\&V database components that will support the LWRS Program, including identifying and populating the NE-KAMS Reactor Systems V\&V database with experimental and numerical benchmark data that will support the LWRS V\&V and UQ assessments.

As a longer term initiative, it is expected that the NE-KAMS effort will work with the American Society of Mechanical Engineers (ASME) V\&V30 standards committee and other interested parties to develop and establish accepted standards, requirements and best practices for Code Scaling, Applicability and Uncertainty (CSAU) [2] and other Best Estimate Plus Uncertainty (BEPU) methods used to systematically quantify reactor systems simulation uncertainty.

\section{References}

1. Dinh, N. T. et. al. A Next Generation of Nuclear Plant System Analysis Codes to Support Risk-Informed Safety Margin Characterization, Proceedings of the 13th International Topical Meeting on Nuclear Reactor Thermal Hydraulics (NURETH13), Kanazawa, Ishikawa-Ken, Japan, 2009.

2. Boyack, B. E. et. al. Quantifying Reactor Safety Margins, papers 1 to 6, Nuclear Engineering and Design, Vol. 119, 1990. 